\title{
RELACIONES INANIMADAS Y CODIFICACIÓN DE RASGOS EN LA FORMA SU/SUS EN EL ESPAÑOL DE LA CIUDAD DE MÉXICO
}

\author{
H. Antonio García Zúñiga \\ Alejandra I. Ortiz Villegas \\ Armando Mora Bustos
}

\section{(c) $\mathbb{P Q \Theta O}$}

Doi: https://doi.org/10.15517/rfl.v46i2.42646

URL: https://revistas.ucr.ac.cr/index.php/filyling/index 



\title{
RELACIONES INANIMADAS Y CODIFICACIÓN DE RASGOS EN LA FORMA SU/SUS EN EL ESPAÑOL DE LA CIUDAD DE MÉXICO
}

\author{
INANIMATED RELATIONS AND FEATURES ENCODING ON \\ THE $S U / S U S$ FORM IN THE SPANISH OF MEXICO CITY
}

\author{
H. Antonio García Zúñiga \\ Alejandra I. Ortiz Villegas \\ Armando Mora Bustos
}

\begin{abstract}
RESUMEN
En este trabajo se plantea que la forma $s u / s u s$ es un determinante con contenido semántico que no codifica exclusivamente posesión. Las construcciones objeto de estudio son del tipo: al pozole se le pone su lechuga, su orégano y su chile para que sepa bueno. Las entidades involucradas en este tipo de construcción son inanimadas, expresan diferentes relaciones semánticas organizadas en, al menos, tres subconjuntos: partes de partes, productos-componentes y de inclusión a partir de referentes creados. La descripción de las frases nominales en donde aparece la forma su/sus se fundamenta en la propuesta de que este determinante es una unidad gramatical que concentra una serie de rasgos, tales como definitud, deixis, posesión, relación, proximidad y empatía. Estos rasgos son consustanciales y coocurren de manera simultánea. Los datos utilizados para ilustrar este hecho de lengua han sido tomados de diferentes corpus.

Palabras clave: posesión; determinante; semántica; sintaxis; español mexicano.
\end{abstract}

\begin{abstract}
The form su/sus is a determiner with semantic content that does not encode possession exclusively. The sentences described are of the type: al pozole se le pone su lechuga, su orégano y su chile para que sepa bueno. The entities involved in this type of sentences are inanimate. These relationships express at least three subsets of inanimate relationships: parts of parts, product-components, and relations of inclusion from created referents. The description of the noun phrases in which the su/ sus form appears is based on the idea that this determiner is a grammatical unit that encodes a set of features, such as definiteness, anaphora/cataphora, possession, relationship, proximity, and empathy. These features are consubstantial and co-occur simultaneously. The data used to illustrate this fact of language have been taken from different sources.
\end{abstract}

Keywords: possession; determiner; semantics; syntax; Mexican Spanish.

\footnotetext{
Mag. Hamlet Antonio García Zúñiga. Profesor investigador de tiempo completo titular B. Museo Nacional de las Culturas del Mundo, Instituto Nacional de Antropología e Historia. México.

Correo electrónico: hamlet_garcia@inah.gob.mx

Mag. Alejandra Itzel Ortiz Villegas. Docente, tutora e investigadora. Instituto de Educación Media Superior. México.

Correo electrónico: aov_26@yahoo.com

Dr. Armando Mora Bustos. Profesor investigador de tiempo completo. Universidad Autónoma Metropolitana, Iztapalapa. México. Correo electrónico: lucioamora@gmail.com
}

Recepción: 08- 10- 19

Aceptación: 11- 02- 20 


\section{Introducción}

La forma su/sus puede caracterizarse como un determinante que posee contenido, es decir, es una unidad gramatical conformada por un conjunto de rasgos a partir de los cuales se interpreta y organiza el sentido de una frase nominal; dichos rasgos son definitud, deixis, posesión, relación, proximidad y empatía. Con base en los resultados de un estudio previo se plantea una exposición más minuciosa de la frase nominal integrada por el determinante su/ sus del español. Este estudio contempla como objetivo mostrar que dicha forma, en ejemplos como los de (1), efectivamente no codifica posesión, sino otra relación semántica entre las entidades involucradas. Cabe destacar que uno de los rasgos que este determinante expresa es la empatía que se establece no solo entre las entidades (empatía interna) sino también con el hablante (empatía externa).

(1) a. A la bicicleta le arreglaron sus pedales, sus ruedas y hasta su cadena.

b. $\quad$ Entonces, ¿le traigo el capuchino con su canela y su espuma?

c. Me encantan las enchiladas con su quesito y su crema.

(OMG)

Si bien no forma parte de los objetivos de este trabajo retomar las discusiones sobre la etiqueta de la categoría determinante, vale la pena destacar algunos aspectos básicos de estas para elaborar una descripción del fenómeno que aquí se aborda. Aquí se considera que los determinantes su/sus forman parte de las categorías funcionales, en el sentido de que establecen inventarios cerrados, además de que sus funciones son estrictamente gramaticales. En este estudio se entiende la etiqueta de determinante como una posición en la estructura gramatical que se llena con varios elementos (categorías), los cuales especifican los rasgos sobre cómo se debe identificar el referente de un sustantivo particular (o, en algunos casos, la clase de relación que tiene este con algún tipo de entidad). Los determinantes incluyen artículos, demostrativos, pronombres posesivos, algunas formas interrogativas, pronombres, etc. (Payne, 2006). Como la mayoría de las categorías funcionales, es una unidad prosódicamente no acentuada y posee un contenido funcional, en el sentido de que no denotan seres o entidades.

Si bien en la literatura hay un acuerdo generalizado sobre la función modificadora del determinante sobre el núcleo nominal, poco se señala sobre este carácter modificador. Por un lado, Van Valin y LaPolla (1997) mencionan que el determinante modifica el sustantivo en cuanto a sus rasgos referenciales. Por otra parte, Dryer (2007, pp. 152-162) señala que en algunas lenguas, como varias de las de Filipinas, las frases nominales aparecen con determinantes que codifican una variedad de propiedades gramaticales, las cuales interactúan con el sistema que da cuenta de la estructura de la información. Hay que reconocer que en esta descripción Dryer (2007) no hace alusión al tipo de propiedades gramaticales que se codifican en el determinante. Bosque y Gutiérrez-Rexach (2008), por su parte, apuntan que los determinantes, como indica su etiqueta categorial, "determinan" o "especifican" la referencia del nombre sobre el que inciden, es decir, precisan su cantidad, su referencia u otras propiedades relacionadas con estas, como son la posesión, anáfora/catáfora, etcétera.

Nótese que, en este sentido, la forma su/sus determina la referencia del nominal que acompaña y expresa la posesión entre esa entidad y una tercera persona, o bien, otro tipo de relación semántica. En específico, en contextos gramaticales en los que las entidades que expresan los nominales sean de una naturaleza inanimada se presenta una relación que remite a contenidos, sustancias, partes, productos y materias. Como se mostrará más adelante, todas estas relaciones 
pertenecen al mismo dominio semántico. El grupo de rasgos mencionados (definitud, deixis, posesión, relación, proximidad y empatía) tiene la característica de que coocurre simultáneamente en todos los contextos de la frase nominal. Lo anterior explica dos cuestiones: en un primer momento, que se manifieste de esta forma el contenido del determinante; en otro, que gracias a esta situación, los rasgos se presentan de manera conjunta.

Este trabajo está organizado de la siguiente manera: en primer lugar, se describe el corpus, el tipo de construcciones objeto de estudio y los antecedentes. En segundo lugar, se presentan las circunstancias lingüísticas que dan cuenta de la complejidad de la forma su/ sus y se explica por qué se considera como un determinante con contenido. En tercer lugar, se examinan las interpretaciones más sobresalientes del concepto de posesión, a partir de esto, se retoma una propuesta en la que se establece que existen dominios semánticos que agrupan distintas relaciones semánticas no eventivas. Esta propuesta funge como marco para el presente estudio. En el penúltimo apartado se explicitan las propiedades sintácticas de este tipo de frases nominales para dar cuenta de la interacción de rasgos que dotan de contenido al determinante su/sus. Finalmente, se detalla el análisis sobre los rasgos del determinante, es decir, sobre la definitud, la anáfora, la relación, la proximidad y la empatía.

El aporte de este trabajo radica en que analiza un hecho de lengua propio de la variedad del español mexicano, en específico el habla de la Ciudad de México, esto es, las frases nominales determinadas por la forma $s u / s u s$ en las que refieren entidades inanimadas. Lo anterior implica un desarrollo lingüístico específico sobre esta estructura lingüística.

\section{Metodología}

La presente investigación se nutre con ejemplos tomados de tres fuentes: la primera corresponde al total de la sección referente a la Ciudad de México del "Proyecto para el Estudio Sociolingüístico del Español de España y de América" (en el texto se citará como PRESEEA). La segunda fuente consiste en material recolectado en el último trimestre del 2017 y durante el 2018 por los autores de este análisis en escuelas públicas de los niveles medio y superior de la entidad político-administrativa mencionada (este material se referirá como $\mathrm{OMG}$ ). La tercera de las fuentes es el "Corpus de Referencia del Español Actual” (mencionado en el trabajo como CREA).

Los primeros dos corpora se emplearon como una guía general del proceso lingüístico que se ha reportado como característico de la Ciudad de México; a saber, el uso común de su/sus en frases nominales como su casa de Juan y isus tacos van con su salsa? En total, los datos de este material ascienden a 792 (283 correspondientes a FN's [frases nominales] con su/sus y 509 FN's marcadas con artículo definido). Por su parte, el corpus integrado por información del CREA se elaboró con el propósito de obtener mayor evidencia de los casos mostrados en (1). La selección de los ejemplos estuvo determinada por la aparición de la forma su/sus dentro de una frase nominal simple o compleja.

Es importante mencionar que la información que aquí se maneja fue balanceada, considerando diferentes tipos de contextos (formal e informal) y géneros (habla espontánea, entrevista, plática, narración, entre otros), con la finalidad de incluir una variedad relativamente amplia de evidencia lingüística que pudiera ser tenida como válida, representativa y no sesgada. De esta forma, la frecuencia, en términos cuantitativos, y el grado de periodicidad en el uso (en un sentido de cotidianeidad o constancia) están en un mismo nivel de representación. 
Aunque en este apartado se han empleado conceptos provenientes de la estadística, por ahora no es parte de nuestro interés realizar un trabajo de índole cuantitativa. Nuestra meta es exclusivamente identificar y mostrar el comportamiento de la estructura en cuestión, además de presentar una propuesta de análisis fundamentada en el funcionalismo (Givón, 2001; Hopper y Thompson, 1980).

\section{Antecedentes}

En este apartado se plantean, en dos sentidos, las generalidades de la propuesta del trabajo. Primero, se describen las circunstancias lingüísticas que permiten entender parte de la complejidad actual que presenta la forma su/sus en diferentes tipos de FN's. Además, se remarca que dicha forma ha redefinido su capacidad de relacionar entidades debido a un desgaste en sus significados referenciales más concretos. Segundo, se destaca la dificultad para enmarcar dentro de una categoría gramatical específica a las llamadas marcas posesivas átonas (ver más adelante). En este sentido, se retoman las hipótesis en las que se considera definirlas como determinantes. A pesar de que a lo largo del texto se insiste en que la perspectiva que se defiende en este trabajo es la de considerar que su/sus es un determinante al que un conjunto variado de rasgos le dan contenido (lo cual permite explicar su amplia gama de usos), es necesario precisar que las obras que en esta sección se explican, por un lado, remiten a la posición sintáctica que su/sus tiene en la FN y, por otro lado, señalan aspectos sintácticos, básicamente, que condicionan su interpretación como determinante.

\subsection{Consideraciones de la forma su/sus en la tradición hispánica}

En los estudios sobre posesión en español se hace la distinción entre dos tipos de marcas: por un lado, la de los posesivos átonos $(\operatorname{mi}(s), \operatorname{tu}(s), \operatorname{su}(s)$, nuestro $(s)$, nuestra $(s)$, vuestro $(s)$, vuestra(s), su(s)) y, por el otro, la de los tónicos (mío(s), mía(s), tuyo $(s)$, tuya $(s)$, suyo(s), suya(s), nuestro(s), nuestra(s), vuestro(s), vuestra(s)). En general, las primeras son posibles antes del sustantivo, mientras que las segundas ocupan una posición sintáctica posterior al núcleo de la frase. Esta investigación trata de forma exclusiva sobre los llamados posesivos átonos $\mathrm{y}$, específicamente, los de tercera persona.

Sobre este tipo de posesivos se ha insistido (Huerta, 2017) en que la posesión nominal ha atravesado un complejo proceso de gramaticalización en el que el pronombre posesivo de tercera persona ha desgastado sus significados referenciales más concretos para dar paso a la creación de significados más abstractos, sin perder los referenciales, con lo que se ha visto obligado a redefinir la capacidad que tiene de relacionar entidades. Este cambio, además, generó un progresivo debilitamiento del valor de control de las entidades codificadas por el posesivo, algo que, sin duda, se requiere en las relaciones posesivas prototípicas.

Existe suficiente evidencia para señalar que lo anterior permitió construcciones, en principio, extrañas, tales como su casa de Juan (ver Company y Huerta, 2017, p. 180). Esta FN presenta una estructura peculiar, en la que destaca su comportamiento poco posesivo. Lo más atípico es que esta construcción muestra una preferencia por poseídos humanos, lo cual conlleva una nivelación de poseído y poseedor y, por tanto, una aminoración de la asimetría requerida en una relación posesiva (Company y Huerta, 2017). 
Como se puede observar, los problemas que existen en torno a las marcas posesivas átonas se sitúan en la semántica de la frase; esto es en la naturaleza referencial del poseído. Sin embargo, hay datos en español que muestran que estos problemas también se dan en lo que podría denominarse semántica de la relación, es decir, en la identificación del poseedor. Este es el caso de los ejemplos de la presente propuesta. Con base en la información que se dispone, se ofrecen argumentos sólidos para poner en duda los rasgos de un poseedor y para concebir como determinante la marca su/sus.

Otro de los problemas que están relacionados con las marcas posesivas átonas es el de su adscripción categorial. Así, el debate se ha centrado en establecer si se deben considerar pronombres, adjetivos, pronombres-adjetivos o determinantes. Según la Nueva Gramática de la Lengua Española (NGLE, 2009), los posesivos son una clase transversal, puesto que exhiben propiedades gramaticales que, por una parte, los asimilan a la clase de los determinantes, en tanto que ocupan en la frase nominal la misma posición inicial que los artículos, así como los demostrativos y, por otra parte, son una variante de los pronombres personales, ya que poseen rasgos de persona y tienen naturaleza referencial. No obstante, en español, los posesivos no integran un paradigma gramatical homogéneo, por lo que en su delimitación categorial es necesario hacer compatibles propiedades de diferentes clases de palabras (Company y Huerta, 2017).

Sobre su clasificación como determinante se puede decir lo siguiente. Lenz (1935), Leonetti (1990, 1999), Company (1991, 2001), Lyons (1993), Eberenz (2000) y Jiménez (2006), analizan los posesivos átonos como determinantes, ya que dan al sintagma nominal interpretación definida, como los artículos; son unidades cuyos rasgos semánticos permiten ubicar una referencia en la esfera del conocimiento del interlocutor a través del parámetro de posesión. A esto se añade, según Picallo y Rigau (1999), que dicho posesivo no solo ocupa la posición de determinante, sino que, de hecho, actúa como tal. Por esto se reconocen ciertas restricciones, por ejemplo, estas autoras recuperan el concepto de "efecto de definitud" para explicar casos como nuestra amiga que siempre viene a visitarnos, mi cuadro más original, sus tazas de porcelana y tu prima de Zaragoza, donde puede haber un contraste con otros modificadores restrictivos (Picallo y Rigau, 1999, p. 978). Asimismo, el posesivo prenominal, a pesar de aparecer en posición de determinante, no legitima un sintagma nominal con núcleo elíptico: *su coche pequeño y mi grande. En otros casos (Picallo y Rigau, 2017), las frases nominales en plural pueden provocar una interpretación distributiva del posesivo (los alumnos repasaban sus apuntes).

\subsection{Su/sus, un determinante con contenido}

Esta clase sintáctica se antepone al sustantivo, esto es, lo determina. Constituye un paradigma formado por artículos, demostrativos, posesivos, cuantificadores (indefinidos y numerales), relativos, exclamativos e interrogativos (NGLE, 2009). El planteamiento de Mora, Ortiz y García (2019) es estrictamente descriptivo y está fundamentado en la gramática funcional (Hopper y Thompson, 1980; Comrie, 1981; Shopen, 1985; Givón, 2001). La idea de que los determinantes codifican rasgos no es nueva, de hecho, ha sido retomada del modelo de la gramática generativa. En este sentido, Ghomeshi, Paul y Wiltschko (2009) muestran que los rasgos que se codifican varían en contenido y distribución. 
Mora, Ortiz y García (2019) plantean que la forma su/sus expresa los rasgos presentados en (2).

(2) Definitud

Deixis (anáfora/catáfora)

Posesión

Relación

Proximidad (positiva/negativa)

Empatía

Como ya se ha aludido, estos rasgos son inherentes al determinante su/sus. Lo que se quiere mostrar en este trabajo es que se actualizan en cada contexto gramatical. Esto aunado al hecho de que coocurren y covarían extensivamente. A partir de estos rasgos, en construcciones como las de (3), el determinante su/sus debe ser interpretado de manera particular.

(3) a. Las flores tornasoladas del dobladillo de su vestido iban a invadir mi existencia.

(CREA)

b. Los habitantes de esta isla en realidad nunca mueren porque cuando revientan comienzan [...] a comerse a sí mismos: se comen sus manos, su estómago, su hígado.

(CREA)

c. $\quad$ En esa esquina siempre espera su taxi.

(OMG)

d. Una mujer de dicha confesión reclama porque en su iglesia le discriminan en lo que respecta al acceso a posiciones de autoridad.

(CREA)

e. Veracruz y su plaza y su palacio municipal que parecía hecho de terrones de azúcar.

(CREA)

Las frases nominales que aparecen introducidas por la forma su/sus se caracterizan por un conjunto de rasgos propios de ese determinante. Por un lado, los rasgos que se exhiben en su vestido (3a) son definitud, deixis y posesión, mientras que en (3b) sus manos, su estómago y su hígado, por referir a entidades que forman partes del cuerpo, están presentes los rasgos de definitud, deixis y de relación. Por otro lado, en su taxi (3c) queda claro que no existe una relación semántica posesiva entre la entidad referida en la tercera persona y la entidad expresada en la frase nominal su taxi, sino que a partir de la repetición o frecuencia de una acción (en esa esquina siempre espera el taxi) se genera un vínculo entre la entidad referida y el hablante, así que el rasgo que destaca, además de la definitud y la deixis, es la proximidad. Por último, en (3d) y (3e) se activan los mismos rasgos: la definitud, la deixis, así como la empatía. Sin embargo, esta última es generada por la percepción que hay del hablante hacia la entidad referida, como también se puede observar en la frase nominal su iglesia (3d) o la percepción de cercanía entre las entidades mismas, como Veracruz y su plaza (3e).

En este sentido, cabe destacar que de las frases nominales antes mencionadas solo se codifica una relación de posesión en (3a); en todas las demás (de 3b a 3e), las relaciones semánticas son de diferente naturaleza y, por ello, los rasgos que se destacan en las frases 
nominales son de distinta índole. En suma, para Mora, Ortiz y García (2019) es indispensable precisar la caracterización semántico-sintáctica de los rasgos asociados a la categoría de determinante.

\section{Propiedades semánticas}

Como se ha mencionado, la forma su/sus codifica un conjunto de rasgos mediante los cuales expresa referentes definidos (4a), generalmente mencionados en el discurso (4b). De igual manera, expresa relaciones semánticas diversas como posesión (4c), o bien, otros tipos de relaciones como parte-todo (4d), parentesco (4e) y contenedores (4f). Por último, también puede expresar los rasgos de proximidad (4g), de empatía, a partir de la actitud que tiene el hablante (4h) o de una empatía interna generada entre las entidades mismas (4i).

(4) a. Espera para 1997 la publicación de su libro.

b. El problema de su vida era que los hombres se enamoraban de "Gilda", su personaje más glamoroso del cine.

c. Como reconoció José Córdoba en su artículo "La reforma económica de México".

d. Tóquelos con la punta de su dedo como si fueran las cuerdas de un arpa.

e. A cuatro metros está la tumba de su primo, Gustavo de Jesús Rivero, su compañero de siempre.

f. De la ropa en su cajón y de las cuentas claras.

g. De perfiles humanos que inspiran sólida confianza en su actuación.

h. Carolina quedó embarazada [...] un día su luna no brilló en el horizonte mensual.

i. Todo era nuestro en el pueblo: sus calles empedradas, sus casas de adobe y cal, su viento transparente y libre.

(CREA)

Es preciso aclarar que este determinante en algunos contextos no expresa posesión, como podemos notar en las construcciones de (5). Esa forma introduce partes de objetos (5a) o entidades que perfilan una relación empática entre ellas, es decir, se perciben como cercanas la carne y su tortilla en (5b), así como la pancita y su caldo en (5c).
a. Al lado, el clavecín con su tapa abierta.
b. El general Suárez era tan simple como una carne con su tortilla de harina.
c. Luego se añaden la pancita con su caldo colado, los garbanzos y el epazote.

(CREA)

El concepto de posesión se ha planteado, tradicionalmente, en términos binarios como alienable e inalienable. Sin embargo, en este trabajo se retoma la perspectiva de un conjunto de relaciones semánticas, las cuales se organizan en cuatro dominios con rasgos caracterizadores como la cohesión de las entidades, la concepción de la unión entre estas y la animicidad de las entidades involucradas (Arellanes, Ortiz, Mora y García, 2014; Ortiz, Mora, Arellanes y García, 2014; Ortiz Villegas, 2015, 2016; Mora, Ortiz y García, 2019).

En el primero de los dominios se establecen las relaciones que existen entre una entidad animada y otra inanimada; tal es el caso de la posesión (6) y meronimia (7), especialmente cuando las partes del cuerpo se ven involucradas. 
(6) a. Llevaba en su maleta, su ropa, sus pantalones, sus blusas, su maquillaje y los zapatos que le habían regalado en su cumpleaños.

b. Revisé sus tareas y es increíble su desorganización.

c. Compró todo para su nueva casa.

$(\mathrm{OMG})$

(7) a. Al muertito le acomodaron su pelo, su bigote, su barba, sus uñas.

b. Mi niña se veía bien bonita con su flequito y su mochilita en su primer día de clases.

c. Iba con su cabello bien peinado.

$(\mathrm{OMG})$

Por su parte, en el segundo dominio se agrupan aquellas relaciones que se forman entre dos entidades inanimadas como en (8). En este dominio tienen cabida las partes de partes (8a), las porciones (8b) y las materias o sustancias (8c).

(8) a. El tic tac de relojes que marcaban distintas horas según sus manecillas.

b. Este es el embuste de los ricos para que los pobres no les coman su trozo de pastel.

c. Para repoblar con mezclas de árboles de especies apreciadas por su madera.

(CREA)

En el siguiente dominio las relaciones semánticas se caracterizan por establecerse entre entidades humanas, es por eso que refieren relaciones sociales consanguíneas o no consanguíneas (9). En el último dominio se establecen relaciones entre un tema y una locación (10).

(9) a. Totalmente desinformado de las andanzas de su hermano.

b. Su abuela lo llevaba a una esquina donde un tipo vendía barquillos.

c. Si algo decía, la misma suerte de su patrona iba a correr ella.

(CREA)

(10) a. Cuando regresa disfruta su tequila jalisciense.

b. Presumiendo llegó con su plata de Taxco.

(OMG)

c. Comitán los recibió con su clima tibio y sus campos florecidos.

De esta manera, se entiende el concepto de posesión en términos de pertenencia que se caracteriza por una entidad [+/-animada] que forma parte del dominio del poseedor, preferentemente humano (Seiler, 1981; Heine, 1997a, 1997b; Taylor, 1999).

Dicho esto, es necesario aclarar que las construcciones de (11) no cumplen con los rasgos propios de la posesión (poseedor +/- animado, posesión inanimada, posesión entendida en términos de 'pertenencia') y, por ello, no forman parte del primer dominio. Por el contrario, las entidades involucradas son entidades inanimadas del tipo del segundo dominio. En específico, estas relaciones expresan al menos tres subconjuntos de relaciones inanimadas, entre ellas destacan: I) partes de partes (11a), las uñas forman parte de un entorno intermedio, en este caso, las manos; II) productos-componentes (11b), en donde interactúan entidades que conforman un producto, la guayaba, la canela, las manzanas, las pasitas y el piloncillo son necesarios para el producto ponche, y III) relaciones de inclusión a partir de referentes creados 
(11c), el nuevo vínculo que se establece entre las posadas y el ponche o las piñatas se genera a partir de la percepción de cercanía entre esas entidades.

(11) a. Siempre trae las manos con sus uñas bien pintaditas.

b. $\quad$ Al ponche se le pone su guayaba, su canela, sus manzanas, sus pasitas o ciruela pasa y su piloncillo.

c. $\quad$ A mí me gustan las posadas, con su ponche y sus piñatas ya se anima la cosa.

(OMG)

Este tipo de construcciones se caracteriza por I) involucrar entidades inanimadas, II) establecer relaciones internas entre las entidades y III) establecer un principio de cercanía entre las entidades involucradas.

El planteamiento que aquí se hace es similar al de Eguren (2018) en el sentido de que identifica y califica como no canónicas a las FN's que tienen como determinante la forma su/ sus. Sin embargo, este autor establece que existe una relación intrínseca entre el poseedor y el poseído, como se ejemplifica en (12). En otros términos, dicho autor reconoce una relación de posesión en esas frases nominales, la cual se establece a partir del punto de vista del hablante. A esto él lo denomina "posesivos prenominales no canónicos evaluativos".
a. El apartamento tiene su cocina y su baño.
b. La casita tenía su tejadito.
c. El coche ya tiene sus años.

(Eguren, 2018, pp. 7-8)

En este sentido, lo que él llama posesivo puede ser omitido o sustituido, como se puede ver en (13).
a. $\quad$ El apartamento tiene cocina y baño.
b. La casita tenía tejadito.
c. El coche ya tiene años.

Es importante resaltar que, desde la perspectiva que aquí se presenta, los casos de (12) no pueden ser llamados posesivos porque, como se vio, no cumplen con las características semánticas propias de la posesión, por lo que la construcción debe ser interpretada dependiendo de los rasgos del determinante. Sin embargo, se coincide con Eguren (2018) en que pueden ser omitidos, o bien, sustituidos por otro determinante dentro de esa frase nominal. De esta manera, si la frase nominal se encuentra modificada por el determinante su/sus, se entiende que codifica los rasgos mostrados en (2). En (14) se repiten estos rasgos, con excepción del de posesión, ya que se pretende destacar la imposibilidad de que este rasgo se active en las construcciones que son objeto de este estudio.

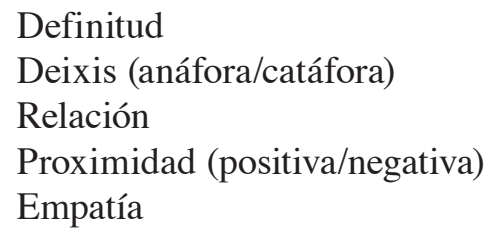

Ahora bien, no debe perderse de vista que construcciones como las de (15) pertenecen al segundo dominio, ya que involucran entidades inanimadas. Aquí se encuentran subclases como las que se mencionaron anteriormente, partes de partes (15a), productos-componentes (15b) y relaciones de inclusión que no son obligatorias (15c). 
(15) a. Hizo cita para sus pestañas, necesita retoque.

b. Puedes preparar una conga sin alcohol mezclando los tres jugos y al final su chorrito de granadina.

c. [...] roscas, pero de las que comen en México [...] no como las de acá que nada más traen su azúcar glas.

(OMG)

Estas frases nominales se caracterizan por mantener relaciones internas y por establecer un principio de cercanía entre ellas. Dicho esto, se reconoce que hay diferentes relaciones semánticas organizadas en distintos dominios semánticos, caracterizados según el tipo de entidades y la cohesión que exista entre ellas. Dados estos fundamentos, en tales contextos, no es posible interpretar la forma su/sus como una marca propiamente posesiva.

\section{Propiedades sintácticas}

Como se dijo anteriormente, la forma su/sus ocupa la posición propia de los determinantes. Este determinante genera un efecto de definitud en la frase nominal en la que aparece, ya que explicita una denotación de una entidad definida, similar a la que generan los artículos. En ese sentido, funciona como un modificador restrictivo; pero, además, en ciertos contextos, esta forma no explicita a una frase nominal con núcleo elidido y no recupera la referencia del poseedor, sino que aparece como una forma que codifica un sentido enfático.

Las frases nominales de (16) están formadas por el proclítico su/sus y su respectivo núcleo nominal. Estos clíticos, de manera similar a lo que ocurre con los artículos de (17) y los demostrativos de (18), se denominan determinantes. Nótese que la presencia de un determinante está condicionada por diversos factores gramaticales: semántica del verbo o del nominal, y el estatus de la FN como relación gramatical o adjunto.

(16) a. Se me antoja una concha así con su natita encima.

b. Los romeritos son muy laboriosos, se lavan, se tiene el mole preparado, se desinfectan,sustortillas (sic)decamarónsehacenaparte, sele pone suhuevoaparte y sus papitas de las chiquitas y sus nopales de los chiquitos.

(17) a. Se me antoja una concha así con la natita encima.

b. Los romeritos son muy laboriosos, se lavan, se tiene el mole preparado, se desinfectan, las tortillas (sic) de camarón se hacen aparte, se le pone el huevo aparte y las papitas de las chiquitas y los nopales de los chiquitos.

(18) a. Se me antoja una concha así con esalesta/aquella natita encima.

b. Los romeritos son muy laboriosos, se lavan, se tiene el mole preparado, se desinfectan, estas/esas/aquellas tortillas (sic) de camarón se hacen aparte, se le pone eselestelaquel huevo aparte y estas/esas/aquellas papitas de las chiquitas y esos/estos/aquellos nopales de los chiquitos.

(OMG)

En las frases nominales de las construcciones de (16), la forma su/sus puede remplazarse por los artículos la/las, el/los, como en (17), y por los demostrativos ese/estelaquel, esos/estos/ aquellos, en (18). Estructuralmente, esto indica que estas categorías son intercambiables y multifuncionales. No obstante, la idea que se plantea a lo largo de este trabajo gira en torno al hecho de que la forma su/sus codifica un conjunto de rasgos, definitud, anáfora/catáfora, 
relación, proximidad y empatía, de modo que el determinante su/sus se considera una categoría funcional que codifica un conjunto de propiedades gramaticales.

En las frases nominales de (19a), sus tamalitos y (19b), sus chiles y su mayonesa, la forma su/sus no expresa un sentido de posesión dado que esas construcciones no pertenecen a ese dominio semántico, más bien remite a una relación de conjunto, es decir, sus tamalitos se encuentran relacionados con atole, mientras que, por su parte, sus chiles y su mayonesa se relacionan con sándwich. En este tipo de construcción, la referencia anafórica está activa y dentro del discurso se recupera la relación que existe entre los elementos del conjunto, así como entre la entidad contenedora y sus respectivos ingredientes.
a. Les dan el primer día atole con sus tamalitos.
b. Le pusieron al sándwich aguacate, sus chiles, su mayonesa.

(OMG)

Con respecto al rasgo de la referencia, Givón (2001) señala que los nominales modificados por este determinante obligatoriamente son definidos y expresan un tipo específico de referencia deíctica anafórica. El sentido de esta apreciación es más notorio en las frases nominales analizadas en este trabajo. En (20), la forma su/sus se remplaza por los artículos indefinido y definido. En los dos casos se desvirtúa el sentido que expresa la frase nominal. Lo que esto revela es que la forma su/sus no se puede remplazar en todos los contextos por un artículo indefinido.
a. Les dan el primer día atole con unos/los tamalitos.
b. Le pusieron al sándwich aguacate, unos/los chiles, ?? una/la mayonesa.

(OMG)

Los rasgos codificados en el determinante $s u / s u s$ tienen la misma naturaleza gramatical. Todos los rasgos exhibidos en esta forma son intrínsecos y, por lo tanto, obligatorios. Estos coaparecen y coocurren libremente, además de que están correlacionados directamente con la expresión discursiva. La prominencia dentro de los rasgos no solo es conceptual sino configuracional. Esto implica que la activación de los rasgos del determinante está asociada a la naturaleza semántica del núcleo y al contexto situacional en el que una expresión es emitida, como en (21). La definitud se complementa con la anáfora o la catáfora, en tanto que la relación, la proximidad y la empatía son consustanciales.

a. $\quad$ No se preocupen, cada vez que preparo ceviche le pongo su jitomate, su limón y su ajo.

(OMG)

b. Ha traído un maletín con sus herramientas de plomero.

(CREA)

Sintácticamente, la forma su/sus es un modificador con contenido que coaparece con núcleos nominales que denotan diferentes significados. Este determinante tiene la propiedad de aparecer en contextos gramaticales en donde los rasgos que codifica se activan de manera consustancial.

\section{Rasgos del determinante su/sus}

La premisa que se ha mantenido en este trabajo está enfocada en el hecho de que la forma su/sus, que aparece en las construcciones objeto de estudio, no codifica el rasgo de posesión 
dado que las entidades involucradas se relacionan semánticamente de distinta forma. Como se ha dicho, la justificación de este hecho se ha fundamentado en que este determinante codifica los rasgos de definitud, anáfora/catáfora, relación, proximidad y empatía. La caracterización de los rasgos se ha generado a partir de la multiplicidad de contextos gramaticales en donde aparece el proclítico su/sus. Tras esta diversidad contextual, en otros estudios (Picallo y Rigau, 1999; Huerta, 2017; Company y Huerta, 2017; Eguren, 2018) se han presentado propuestas para dar cuenta de la naturaleza de esta forma. Aunque en este trabajo no se establece una discusión profunda sobre los detalles de dicha bibliografía, se puede decir, por ejemplo, que Picallo y Rigau (1999) señalan que la proximidad de sentido de los posesivos átonos y tónicos puede verse en la polifuncionalidad de los usos (vocativos, interjecciones y formas exclamativas); o bien, Eguren (2018), más que referir a la "polifuncionalidad" considera que la forma su/sus aparece duplicada, así se establece, desde el punto de vista del hablante, una relación intrínseca entre el "poseedor" y el "poseído". En resumen, se ha planteado una tipología más amplia de los usos y las interpretaciones de los llamados posesivos. De esta forma, tanto el uso como la interpretación determinan las etiquetas con las que se conocen a estos "tipos" linguiísticos, ya sea como proximidad de sentido, interpretación caracterizadora o de estereotipo, y posesivos prenominales no canónicos evaluativos. Lo interesante es que estas explicaciones se acercan, en alguna medida, al concepto de algunos de los rasgos que aquí se proponen. No obstante, hay que señalar que la nomenclatura que se maneja en este trabajo no se refiere a las diferentes interpretaciones que pueden tener las marcas posesivas; más bien, remite a rasgos particulares, lo cuales, en conjunto, dan el sentido de determinante a la forma su/sus.

\subsection{Definitud y anáfora/catáfora}

Aquí se asume que la forma su/sus, como en (22), es inherentemente definida, codifica un sentido de definitud o especificidad y, en consecuencia, genera un alto grado de referencia del núcleo. La referencia generada por el pronombre en las frases nominales su nievecita y su fumarola de (22a), con sus actores y sus argumentos de (22b), su llanta y su birlo de seguridad de (22c), y su discusión de (22d), presenta un alto grado de especificidad. En parte, este carácter está generado por la referencia deíctica que se encuentra anclada en la entidad con la que está relacionada. Las frases nominales con la forma sus/sus, como en el ejemplo (22), están en una relación de dependencia con respecto a: el Popo de (22), las telenovelas de (22b), mi coche de (22c), y la iniciativa de ley de (22d).

(22) a. Mira qué bonito está el Popo con su nievecita y su fumarola.

b. Las telenovelas brasileñas son muy buenas, con sus actores, sus argumentos.

c. Anoche se le ponchó su llanta a mi coche cuando quise cambiarla no encontré su birlo de seguridad.

(OMG)

d. La iniciativa de ley que debe entrar para su discusión al Congreso de la Unión. (CREA)

Mora, Ortiz y García (2019) consideran que las propiedades de la definitud son la familiaridad y la anáfora/catáfora. Dentro de la perspectiva de la familiaridad, el referente de una frase nominal definida es conocido por los interlocutores del acto de habla. Este referente ya debe de haber sido mencionado en el discurso. En sentido amplio, la definitud 
de una entidad está relacionada con el ámbito anafórico del referente en cuestión y con el conocimiento general que se tenga, por parte de los interlocutores, sobre este referente. El determinante su/sus, de manera obligatoria, liga las anáforas/catáforas. En el ejemplo de (23), la frase sus especias hace referencia a un tipo de barbacoa. En este contexto la frase con sus especias aparece coordinada con una frase nominal modificada por un artículo, el chile. Las FN's modificadas por su/sus son altamente definidas. El grado de definitud está asociado con el hecho de que solo se correlaciona con una sola unidad en el universo del discurso.

A: ¿Y cómo la hacen? ¿Nada más, así como tipo barbacoa ${ }_{i}$ ?

B: $\quad$ Ajá, se prepara el chile ${ }_{i}$ bien y con todas sus especias en/j $_{\mathrm{j}}$ y se enchila la carne y ya se pone en los hornos.

(Mora, Ortiz y García, 2019, p. 26)

Las frases nominales, como sus especias de (23), presentan un aparente problema de correferencia, el cual se asocia a un sentido de ambigüedad del determinante su/sus. La referencia que establece dicha forma puede estar dentro del ámbito del acto de habla (referencia exofórica) o dentro del discurso (endofórica). En el primer caso $s u s_{\mathrm{j}}$ estaría haciendo correferencia con una tercera persona quien sería la poseedora de las especias, mientras que en el segundo $s u s_{\mathrm{i}}$ hace correferencia con el referente chile $_{\mathrm{i}}$ y barbacoa $_{\mathrm{i}}$. Con respecto a este hecho se debe señalar que, en los ejemplos encontrados en el corpus, la interpretación de la correferencia exofórica no es posible.

Con todo lo expuesto se puede observar que los rasgos de definitud y anáfora/catáfora están correlacionados, por lo que son consustanciales, en un sentido de restricción, de la referencia de la entidad expresada en el nominal nuclear. La definitud depende del rasgo de familiaridad, por lo que el referente del nominal debe estar bajo el conocimiento de los interlocutores, de tal manera que la referencia anafórica/catafórica sea fácil de reconocer o identificar.

\subsection{Relación}

Este rasgo establece una relación inherente entre las entidades y se caracteriza por estar asociado a relaciones semánticas de diversa índole, de las que destacan cuatro tipos: I) parte-todo animado, II) parte-todo inanimado, III) relaciones sociales y IV) relaciones que conforman productos. Las primeras se identifican por la referencia a partes del cuerpo, humano o animal, como las construcciones de (24a) y (24b), su brazo y su patita, respectivamente; mientras que en las del segundo tipo, el todo debe ser inanimado forzosamente, como en (24c), en donde su techo es una parte del todo casa. En este segundo tipo también se sub-agrupan relaciones que involucran partes de partes como su vidrio (24d). En el tercer tipo se encuentran relaciones sociales como las de parentesco por consanguinidad transgeneracional (24e) o intergeneracional (24f), por afinidad (24g), por adopción o por algún vínculo emocional o de jerarquía que se establezca entre dos personas (24h). Por último, en el cuarto tipo, si los ingredientes son necesarios para la elaboración del producto final, también se establece una relación obligatoria (24i).

(24) a. Sóbale su brazo, se pegó bien duro.

b. $\quad$ lo traje porque trae lastimada su patita.

c. Con lo del temblor se le cayó su techo, tuvieron que ponerle lámina a su casa.

d. Con todo el alboroto de anoche le rompieron su vidrio. 
e. $\quad$ Uy, su abuelo lo cuida mucho.

f. Su hermano ya salió de la prepa.

g. Mírala, ahí viene con su suegra.

h. $\quad$ Tiene que andar a las vivas porque si no su patrón lo regaña.

i. $\quad$ El alambre lleva su pimiento, su cebolla y pollo o bistec, lo que quiera...

(OMG)

Del conjunto de relaciones ejemplificadas en (24) forman parte de este estudio las que se repiten en (25), las cuales se caracterizan por tener un todo inanimado (25a), partes de otras partes (25b) e ingredientes obligatorios para la elaboración de un producto (25c), las relaciones parte-todo animado y las relaciones sociales quedan fuera de los límites de este trabajo.
a. $\quad$ Con lo del temblor se le cayó su techo, tuvieron que ponerle lámina a su casa.
b. $\quad$ Con todo el alboroto de anoche le rompieron su vidrio.
c. El alambre lleva su pimiento, su cebolla y pollo o bistec, lo que quiera...

$(\mathrm{OMG})$

En los ejemplos de (25) se aprecia que los rasgos que están codificados en la forma su/sus coaparecen, expresan referentes definidos, están mencionados en el discurso y generan relaciones semánticas específicas.

\subsection{Proximidad}

La proximidad es un rasgo que se caracteriza por establecer una relación conceptual, una cercanía espacial y/o temporal entre las entidades. Este concepto se delimita a partir de la intención o el deseo de acercamiento entre las mismas. El rasgo se delinea a partir de tres clases: I) la existencia de una entidad parcial, es decir, que no existe en su totalidad, II) la presencia de una entidad que ya existe y que se concibe como próxima o cercana y III) la existencia de una entidad en un plano abstracto. Estos rasgos se encuentran ilustrados respectivamente en los ejemplos de (26).

a. Quiero su tarea bien hecha, nada de entregármela rayoneada.

(OMG)

b. Los robots japoneses son los mejores por su tecnología y su parecido con los humanos.

c. Ese es su don, dibuja muy bien.

(CREA)

(OMG)

Las entidades involucradas se relacionan total o parcialmente, como se explica a continuación: a) la entidad existe en el mundo parcialmente y puede formar parte del dominio de la otra entidad, b) existe en el mundo de forma total y puede formar parte del dominio de la otra entidad, c) existe en el mundo totalmente y nunca formará parte del dominio de la otra entidad y d) existe en tanto que los valores universales (positivos o negativos) no sean inherentes al individuo (Mora, Ortiz y García, 2019).

En el ejemplo de (26b) se ilustra la proximidad entre entidades expresadas en las frases nominales los robots japoneses y su tecnología, así contextualmente el referente de la entidad existe en el universo del discurso, mientras que el sentido expresado por la forma $s u /$ sus codifica el rasgo de proximidad. 


\subsection{Empatía}

Recuérdese que las construcciones no posesivas que se analizan en el presente estudio se caracterizan por este rasgo. En sí, la empatía (Moya-Albiol, Herrero y Bernal, 2010) consiste en la identificación mental y afectiva que se genera entre un individuo y una entidad, la cual se encuentra marcada por la forma su/sus. En otras palabras, con este rasgo se identifica al referente del nominal como una entidad íntima o cercana, casi propia. Para que la empatía se logre las entidades se perciben en términos positivos, a través de un vínculo afectivo o afín.

Este rasgo se clasifica de acuerdo con la relación que se tenga entre la entidad y el hablante, como en (27a), o bien, entre dos entidades inanimadas, como en (27b); la primera se distingue como empatía externa y la segunda como empatía interna.

a. Amé "La casa de las flores" por el tonito de su actriz.

b. Al preparar el pollo le podemos poner lo que viene siendo su sal y su pimienta.

(OMG)

La relación afectiva que se establece entre las frases nominales La casa de las flores y su actriz la hace el hablante, por eso se establece una empatía externa, mientras que en la empatía interna existe una relación de sintonía o de afinidad entre las entidades como el pollo y su sal y su pimienta. En principio, este rasgo podría considerarse equivalente a los posesivos prenominales no canónicos evaluativos de Eguren (2018). Sin embargo, esto sería cierto únicamente en los casos en los que la entidad susceptible de considerar algo como cercano a su dominio sea de una naturaleza referencial animada.

Los rasgos descritos hasta ahora forman parte del contenido del determinante su/ sus. Es importante remarcar que, en los ejemplos descritos en el presente trabajo, el rasgo de posesión no se codifica.

\section{Conclusiones}

En el planteamiento desarrollado, se han tomado en consideración los estudios de la tradición hispánica en donde se ha planteado que la forma su/sus ocupa la posición de determinante y genera un efecto de definitud en la frase nominal en la que aparece, debido a que explicita una denotación de una entidad definida, de manera parecida a la que generan los artículos. Igualmente, se ha considerado que este pronombre, en algunos contextos, no da cuenta de una frase nominal con núcleo elidido, ya que en algunos casos no recupera la referencia del poseedor. Por esto, más bien, aparece como una forma que codifica un sentido enfático y que destaca rasgos como la empatía que se establece entre las entidades involucradas.

En este trabajo se ha desarrollado la idea de que la forma su/sus, en este tipo de construcciones, no codifica posesión. La cancelación del rasgo de posesión en la forma sul sus se genera en contextos gramaticales en donde las entidades que expresan los nominales son inanimadas. Asimismo, aparece en relaciones que dan cuenta de contenidos, sustancias, partes, productos y materias; todas estas relaciones pertenecen al mismo dominio semántico. Dado que esta forma se realiza como un determinante con contenido, los rasgos codificados, definitud, deixis, proximidad y empatía se activan de manera conjunta. Estos rasgos coocurren de manera simultánea en todos los contextos de la frase nominal.

Semánticamente, las construcciones analizadas no cumplen con los rasgos propios de la posesión (poseedor +/- animado, posesión inanimada, posesión entendida en términos 
de 'pertenencia') dado que las relaciones que se establecen no pertenecen al primer dominio, no hay un poseedor animado que se encuentre involucrado. Las entidades involucradas, en las construcciones estudiadas, son entidades inanimadas del tipo del segundo dominio. En específico, estas relaciones expresan al menos tres subconjuntos de relaciones inanimadas, entre ellas destacan: a) partes de partes, b) productos-componentes y c) relaciones de inclusión a partir de referentes creados.

Sintácticamente, en las frases nominales de las construcciones en donde aparece la forma su/sus puede remplazarse por los artículos y por los demostrativos. Estructuralmente, esto indica que estas categorías son intercambiables y multifuncionales. No obstante, la idea que se plantea en este trabajo gira en torno al hecho de que la forma su/sus debe considerarse como un determinante con contenido, es decir, como una categoría funcional que codifica un conjunto de rasgos gramaticales.

El análisis presentado en este artículo abre los horizontes en el ámbito de la lingüística descriptiva por al menos dos razones: la primera, por considerar que las categorías gramaticales clasificadas como cerradas presentan una gramática mucho más compleja de lo que hasta el momento se ha dicho; estas unidades no son discretas, por el contrario, se ha mostrado que son unidades con contenido gramatical, cuyos rasgos se actualizan en cada contexto. La segunda razón referida es por mostrar que en una variedad específica del español se establece una relación empática entre dos entidades inanimadas codificadas en una frase nominal determinada por la forma su/sus.

Así, la complejidad gramatical que presentan los determinantes se convierte en un hecho revelador con respecto al hecho de que la lengua española simplemente es una lengua más dentro del gran entramado de lenguas en el mundo y que, posiblemente, analizar sus rasgos desde una perspectiva tipológica conllevaría simplemente señalar que su comportamiento exótico no es sino una propiedad más dentro de la gran arquitectura de lenguas.

\section{Bibliografía}

Arellanes Arellanes, F., Ortiz Villegas, A., Mora Bustos, A. y García Zúñiga, H. A. (setiembre, 2014). Syntax of the non-eventive semantic relations in four otomanguean languages: amuzgo, mazahua, mazatec and zapotec. Ponencia llevada a cabo en el 6th International Conference Syntax of the World's Languages. Università di Pavia, Italia.

Bosque, I. y Gutiérrez-Rexach, J. (2008). Fundamentos de sintaxis formal. Madrid: Akal.

Company, C. (1991). La frase sustantiva en el español medieval. Cuatro cambios sintácticos. México: Universidad Nacional Autónoma de México.

Company, C. (2001). Gramaticalización, debilitamiento semántico y reanálisis. El posesivo como artículo en la evolución sintáctica del español. Revista de Filología Española, 81(1-2), 49-87.

Company, C. y Huerta, N. (2017). Frases nominales sobrespecificadas encabezadas por un posesivo átono: su casa de Juan, su casa que tiene Juan. En C. Company y N. Huerta (Eds.), La posesión en la lengua española (pp. 177-218). Madrid: Consejo Superior de Investigaciones Científicas.

Comrie, B. (1981). Language Universals and Linguistic Typology. Syntax and Morphology. Chicago: The University of Chicago Press. 
Dryer, M. (2007). Noun phrase structure. En T. Shopen (Ed.), Clause Structure, Language Typology and Syntactic Description (Vol. 2) (pp. 151-205). Cambridge: Cambridge University Press.

Eberenz, R. (2000). El español en el otoño de la Edad Media. Sobre el artículo y los pronombres. Madrid: Gredos.

Eguren, L. (2018). Evaluative prenominal possessives in Spanish. Borealis: An International Journal of Hispanic Linguistics, 7(1), 1-26.

Ghomeshi, J., Paul, I., Wiltschko, M. (2009). Determiners. Amsterdam: John Benjamins Publishing Company.

Givón, T. (2001). Syntax. (Vol. 1). Amsterdam/Philadelphia: John Benjamins Publishing.

Heine, B. (1997a). Cognitive Foundations of Grammar. Oxford: Oxford University Press.

Heine, B. (1997b). Possession: Cognitive Source, Forces, and Grammaticalization. Cambridge: Cambridge University Press.

Hopper, P. y Thompson, S. (1980). Transitivity in Grammar and Discourse. Language, 56(2), 255-299.

Huerta, N. (2017). Posesivos átonos. Sintaxis y semántica. En C. Company y N. Huerta (Eds.), La posesión en la lengua española (pp. 71-131). Madrid: Consejo Superior de Investigaciones Científicas.

Jiménez, T. (2006). El paradigma determinante en español: Origen nominativo, formación y características. Verba, anexo 56.

Lenz, R. (1935). La oración y sus partes. Madrid: Junta para la Ampliación de Estudios e Investigaciones Científicas.

Leonetti, M. (1990). El artículo y la referencia. Madrid: Taurus.

Leonetti, M. (1999). El artículo. En I. Bosque y V. Demonte (Coord.), Gramática descriptiva de la lengua española (Vol. 1) (pp. 787-890). Madrid: Espasa-Calpe.

Lyons, Ch. (1993). El desarrollo de las estructuras posesivas en el español temprano. En R. Penny (Ed.), Actas del Primer Congreso Anglo-Hispano (pp. 215-223). Madrid: Castalia.

Mora, A., Ortiz, A. y García, H. A. (2019). Un determinante con contenido: el caso de su/sus. Iztapalapa Revista de Ciencias Sociales y Humanidades, 86, 11-39.

Moya-Albiol, L., Herrero, N. y Bernal, C. (2010). Bases Neuronales de la empatía. Revista de Neurología, 50(2), 89-100.

Ortiz Villegas, A. (octubre, 2015). La posesión atributiva en mazateco. Ponencia llevada a cabo en el XIII Congreso Nacional de Lingüística. Universidad Autónoma de Chiapas, Chiapas.

Ortiz Villegas, A. (abril, 2016). Sistema posesivo de las partes del cuerpo en mazateco. Ponencia llevada a cabo en el XI Coloquio de Lingüística. Escuela Nacional de Antropología e Historia, Ciudad de México. 
Ortiz Villegas, A., Mora Bustos, A., Arellanes Arellanes, F. y García Zúñiga, H. A. (Abril, 2014). Codificación de relaciones semánticas no eventivas en cuatro lenguas otomangues. Ponencia llevada a cabo en el Coloquio sobre lenguas otomangues y vecinas 6. Biblioteca de Investigación Juan de Córdova, Oaxaca.

Payne, T. (2006). Exploring Language Structure. A Student's Guide. Cambridge: Cambridge University Press.

Picallo, C. y Rigau, G. (1999). El posesivo y las relaciones posesivas. En I. Bosque y V. Demonte (Coord), Gramática descriptiva de la lengua española (Vol. 1) (pp. 9731021). Madrid: Espasa-Calpe.

Picallo, C. y Rigau, G. (2017). La interpretación semántica de los posesivos. En C. Company y N. Huerta (Eds.), La posesión en la lengua española (pp. 267-296). Madrid: Consejo Superior de Investigaciones Científicas.

PRESEEA. (2019). Corpus del Proyecto para el estudio sociolingüístico del español de España y de América. Recuperado de http://preseea.linguas.net

Real Academia Española y Asociación de Academias Americanas. (2009). Nueva Gramática de la Lengua Española. (Vol. 1). Madrid: Espasa.

Real Academia Española. (2019). Corpus de referencia del español actual (CREA). Recuperado de http://www.rae.es/

Seiler, H. (1981). Possession as an Operational Dimension of Language, 42. Köln: Institut für Sprachwiss, Universität Köln.

Shopen, T. (1985). Language Typology and Syntactic Description. (Vol. 1, 2, 3). Cambridge: Cambridge University Press.

Taylor, J. (1999). Possession. En K. Brown y J. Miller (Eds.), Concise Encyclopedia of Grammatical Categories (pp. 300-303). Oxford: Elsevier Science.

Van Valin, R. y LaPolla, R. (1997). Syntax: structure, meaning and function. Cambridge: Cambridge University Press. 